\title{
Mutación m.3271T>C del gen MT- TL1 en encefalopatía mitocondrial, acidosis láctica y episodios stroke like (MELAS) en edad pediátrica. A propósito de un caso
}

García-Beristain JC¹, Barragán-Pérez EJ²

\begin{abstract}
Resumen
INTRODUCCIÓN: la encefalopatía mitocondrial, acidosis láctica y episodios stroke like (MELAS) es una enfermedad metabólica poco común, que usualmente se presenta con crisis epilépticas y episodios stroke like en personas jóvenes. Se ha visto que 7 a $15 \%$ de los pacientes con MELAS tienen transiciones $\mathrm{T}$ a $\mathrm{C}$ en la posición de nucleótido 3271 (T3271C). La mayoría de los pacientes descritos con esta asociación son de origen japonés. La alteración del estado mental y la esquizofrenia, que en ocasiones puede preceder o acompañar a los episodios stroke-like en MELAS, pueden encontrarse incluso en familiares afectados por la misma mutación en T3271C. Los niños con MELAS tienen amplio espectro de presentaciones clínicas, en las que predominan los episodios stroke like.
\end{abstract}

OBJETIVO: describir los datos clínicos relacionados con la mutación m.3271 >C del gen MT-TL1 en MELAS en un paciente.

CASO CLÍNICO: niña de 11 años de edad en cuya historia había cuadro de enfermedad vascular cerebral con manifestaciones visuales principalmente y posterior deterioro hemodinámico franco con estado de choque, hiperlactatemia y desequilibrio ácido-base severo, lo que permitió determinar la presencia de la mutación $m .3271 T>C$ en heteroplasmia del gen MT-TL1 compatible con MELAS.

CONCLUSIONES: se puede considerar que en los pacientes con alteraciones ácido-base severas e hiperlactatemia franca, que pueda condicionar inestabilidad hemodinámica o estado de choque, se encuentran en relación directa con esta mutación, por lo que en estos pacientes sería necesario un estudio inmediato ante la sospecha, incluyendo la confirmación molecular del mismo en vista de lo agresivo y progresivo del cuadro.

PALABRAS CLAVE: MELAS, encefalopatía, genética, stroke, mutación, acidosis, $m .3271 T>C$, gen.
${ }^{1}$ Adscrito al departamento de neurología.

`Jefe del departamento de neurología.

Hospital Infantil de México Federico Gómez, México.

Recibido: 2 de junio del 2016

Aceptado: 31 de octubre del 2016

Correspondencia

Juan Carlos García Beristain

beristainjc@gmail.com

Este artículo debe citarse como

García-Beristain JC, Barragán-Pérez EJ. Mutación m.3271T>C del gen MT-TL1 en encefalopatía mitocondrial, acidosis láctica y episodios stroke like (MELAS) en edad pediátrica. A propósito de un caso. Acta Pediatr Mex. 2017;38(3):165-174. 


\section{Mutation m.3271T > C in the MT- TL1 gene in Mitochondrial Encephalopathy, Lactic Acidosis and Stroke Like Episodes (MELAS) in pediatric age. Case report.}

\author{
García-Beristain JC ${ }^{1}$, Barragán-Pérez EJ²
}

\begin{abstract}
INTRODUCTION: Mitochondrial Encephalopathy, Lactic Acidosis and Stroke-like episodes (MELAS) is a rare metabolic disease that usually presents with seizures and stroke-like episodes in young people. It been determined that 7 to $15 \%$ of patients with MELAS have transitions $\mathrm{T}$ to $\mathrm{C}$ at nucleotide position 3271 (T3271C). Most patients reported with this association are of Japanese origin. Altered mental status and schizophrenia can sometimes precede or accompany stroke-like episodes in MELAS; they can be found even in families affected by the same mutation T3271C. Children with MELAS have a wide range of clinical presentations, which are predominant Stroke-like episodes.
\end{abstract}

OBJECTIVE: To describe the clinical findings related to $m .3271>C$ mutation of the MT-TL1 gene in MELAS syndrome in this pediatric case.

CLINICAL CASE: A case of an 11 years old female who presents clinical evidence of Cerebral Vascular Accident (CVA) with visual manifestation followed by hemodynamic deterioration, severe shock whit hyperlactatemia and acid-base imbalance. It was determined that there was mutation m.3271T>C heteroplasmy in the MT-TL1 gene compatible with MELAS.

CONCLUSIONS: We believe that patients with stroke, abnormal acid-base and severe hiperlactatemia could lead to hemodynamic instability or shock, in direct relation with this mutation. Therefore these patients must be immediately studied to make the suspected diagnosis. The study must include molecular confirmation before the condition become aggressive and progressive.

KEYWORDS: MELAS; encephalopaty; genetic; stroke; mutation; acidosis; $m .3271 T>C$; gene
${ }^{1}$ Adscrito al departamento de neurología. 2Jefe del departamento de neurología.

Hospital Infantil de México Federico Gómez, México.

Correspondence

Juan Carlos García Beristain beristainjc@gmail.com

\section{INTRODUCCIÓN}

La encefalopatía mitocondrial, acidosis láctica y episodios stroke-like (MELAS) es una enfermedad metabólica poco común, que usualmente se presenta con crisis epilépticas y episodios stroke-like en personas jóvenes. ${ }^{1}$ Fue reportada en 1984 por Pavlakis, quien describió en la literatura a dos 
niños y nueve casos similares con un desarrollo inicial normal, corta estatura, convulsiones y hemiparesias alternantes, además de hemianopsia y sangrado cortical. ${ }^{2}$

Algunos estudios señalan que la prevalencia de miopatía mitocondrial es cercana a 0.58/100,000 en la población total con MELAS como su principal subtipo. En general, los estudios epidemiológicos estiman una prevalencia mínima de 1/5,000 de enfermedad mitocondrial en la población general. ${ }^{3}$

Al ser MELAS el subtipo más dominante de las miopatías mitocondriales y al encontrarse asociado con mayor frecuencia a la mutación A3243G en el $t R N A$ mitocondrial, existen estudios que han señalado la prevalencia de esta mutación por arriba de 1.41/100,000 habitantes en población inglesa adulta y 18.4/100,000 en población pediátrica. ${ }^{3}$

Por otro lado, se ha determinado que 7 a $15 \%$ de los pacientes con MELAS tienen transiciones $\mathrm{T}$ a C en la posición de nucleótido 3271 (T3271C), la mayoría de los pacientes descritos con esta asociación son de origen japonés. ${ }^{2}$

Existen informes de 17 mutaciones en asociación con MELAS, pero sólo algunas han mostrado una asociación, o bien, una expresión patológica; $80 \%$ de los pacientes tienen una transición A o G en la posición del nucleótido 3243 en el gen mitocondrial tRNALeu correspondiente al UUR ( $\mathrm{R}=\mathrm{A}$ o $\mathrm{G}$ ) codones leucina. ${ }^{2}$ Otros estudios mencionan hasta 39 mutaciones de ADN diferentes asociados a MELAS. ${ }^{3}$

La sospecha de MELAS se basa en los hallazgos de los estudios de resonancia magnética nuclear, donde se pueden observar zonas sugerentes de infartos, de las cuales algunas lesiones no se encuentran confinadas a territorios vasculares. ${ }^{2}$ La resonancia magnética es ahora la columna vertebral del diagnóstico junto con otros estudio como biopsia muscular y genéticos. No se cuenta al momento con datos patognomónicos en los estudios de imagen; sin embargo, las lesiones que se consideran como migratorias que no se encuentran bien delimitadas a un territorio arterial específico, como se comentó previamente, serían muy sugerentes de un desorden de tipo metabólico o mitocondrial. ${ }^{1}$

En los estudios en plasma no existen patrones patognomónicos de la enfermedad. Las cifras elevadas de lactato indican metabolismo anaeróbico como dato inespecífico. ${ }^{1}$ Los estudios en líquido céfalo raquídeo son útiles para descartar otras patologías como encefalitis o vasculitis; Los estudios en líquido céfalo raquídeo tanto virológicos como bacteriológicos usualmente son negativos; los niveles de piruvato y lactato usualmente aparecen incrementados, lo cual podría hacer sospechar el diagnóstico inicial. ${ }^{1}$

Otros hallazgos como las cardiomiopatías se han descrito en $20 \%$ de los pacientes con MELAS y la mutación A3243G; sin embargo, no existen informes de cardiomiopatía con la mutación T3271C. Del mismo modo, el retraso en el desarrollo de caracteres sexuales así como la deficiencia parcial de hormona de crecimiento se ha visto asociado en su mayoría a la mutación A3243G. ${ }^{2}$

Como se comentaba previamente la biopsia de músculo es útil para determinar si existe correlación con una patología mitocondrial; se utiliza típicamente el vasto lateral, por lo regular el músculo contiene altos niveles de la mutación en comparación con el plasma. ${ }^{1}$ Las fibras rojas rasgadas con tinción de Gomori son indicativas de enfermedad mitocondrial y pueden encontrarse en MELAS y epilepsia mioclónica con fibras rojas rasgadas. ${ }^{1}$

Adicional a lo comentado otros síntomas agregados a esta entidad pueden ser la cefa- 
lea episódica con vómitos, déficit auditivo y demencia lentamente progresiva junto con esquizofrenia, los cuales puede preceder o acompañar a los episodios stroke-like en MELAS, mismos datos que pueden encontrarse incluso en familiares afectados por la misma mutación en T3271C. ${ }^{2}$ Los niños con MELAS tienen un amplio espectro de presentaciones clínicas en las que predominan los episodios de stroke-like. ${ }^{4}$

La patogénesis de los episodios stroke-like en MELAS no se encuentra bien determinada, pero se ha llegado al consenso de que las lesiones representan edema vasogénico. Existen tres hipótesis principales: la primera que resultaría de un cambio isquémico causado por hipoperfusión resultante de anormalidades vasculares; la segunda, que sería una citopatía generalizada causada por fosforilación oxidativa en las neuronas o glía además de hiperperfusión y edema vasogénico. La tercera, por hiperexcitabilidad neuronal con incremento en la demanda de energía, lo que causa edema vasogénico y necrosis cortical. ${ }^{4}$

De acuerdo a informes epidemiológicos, MELAS se puede dividir en dos subgrupos: la forma juvenil y la del adulto, siendo diferentes las manifestaciones clínicas en cada grupo, diversos estudios consideran a la forma juvenil como la más severa, con más alta mortalidad y más rápida progresión de la enfermedad en comparación a la forma del adulto. ${ }^{3}$

Hasta el momento existe un número limitado de estudios de fenotipos atípicos asociados con m.3271T>C. En particular Suzuki y sus colaboradores en 1996 describieron un paciente con la mutación m.3271T>C, quien únicamente tenía diabetes sin evidencia clínica de alteración cardiaca o cerebral. ${ }^{5}$ Nigashima y sus colaboradores en 2001 describieron a tres pacientes con fenotipo de epilepsia mioclónica con fibras rojas rasgadas caracterizado por neuropatía periférica. Pherson y sus colaboradores en 2005 describieron una niña afectada por artrogriposis distal de nacimiento que tenía un fenotipo MELAS. ${ }^{5}$ Sólo son anecdóticos los informes de asociación con cardiopatía en pacientes con $m .3271 T>C .{ }^{5}$ La relación que hay entre el genotipo y el fenotipo clínico de la encefalomiopatía mitocondrial es compleja e imprecisa. Un mismo tipo de síndrome clínico puede estar asociado con diferentes puntos de mutación en el mismo $m t A D N$. La investigación entre las correlaciones del genotipo y el fenotipo clínico es insuficiente para determinar la patogénesis y las implicaciones en el tratamiento de la encefalomiopatía mitocondrial. ${ }^{6}$ Finalmente, se ha confirmado que en madres e hijos de la misma familia no existe correlación clínica entre la mutación y la severidad clínica. ${ }^{5}$

\section{PRESENTACIÓN DEL CASO}

Niña de 11 años de edad con una prima materna con lupus eritematoso sistémico como único dato heredofamiliar de importancia; perinatales sin datos relevantes para padecimiento actual. Desarrollo psicomotor adecuado. Tuvo varicela a los 6 años de edad; astigmatismo desde los 5 años de edad, por lo que usa anteojos. Niega hospitalizaciones previas, intervenciones quirúrgicas, alergias o transfusiones, era previa sana.

Un mes antes de su primer internamiento inició su padecimiento con cefalea hemicraneal izquierda, pulsátil intensidad 9/10 escala visual análoga de dolor; visión borrosa, náusea, vómito en dos ocasiones acompañado de epistaxis con duración de 24 horas sin presentar mejoría con tratamiento analgésico. Se reportó irritable, agresiva y en ocasiones deprimida con Ilanto fácil. Se realizó dentro de su abordaje electroencefalograma que se reportó normal. Posterior a cuadro inicial fue valorada por un neurólogo pediatra que diagnosticó migraña e inició manejo preventivo, presentando respuesta parcial. Una 
tomografía computarizada de cráneo mostró una probable imagen tumoral occipital izquierda.

Fue referida a nuestro instituto con diagnóstico de astrocitoma de bajo grado occipital izquierdo con historia de un mes de evolución. Llamó la atención la agudeza visual OI 20/30, OD 20/100, campimetría por confrontación alterada en campo temporal derecho; discriminación de colores adecuada, sin compromiso de nervios craneales; fuerza muscular $4 / 5$ de las extremidades inferiores, resto normal.

Se decidió la realización de una resonancia magnética de encéfalo que mostró lesión cortical del lóbulo occipital izquierdo, hipointensa en $\mathrm{T} 1$, hiperintensa en T2 que no comprometía un territorio arterial específico sugestiva de infarto subagudo. (Figura 1), así mismo una angiorresonancia en la que se reportó vasoespasmo bilateral de las arterias cerebrales posteriores de predominio izquierdo. Se inició protocolo de enfermedad cerebro vascular. Los estudios paraclínicos generales fueron normales. El líquido céfalo raquídeo mostró datos citoquímicos y citológicos normales; las serologías virales fueron negativas, al igual que los valores de homocisteína los cuales reportaron como normales; se informa homocigoto mutado normal; factor $\mathrm{V}$ Leiden normal. Mutación protrombina normal. Estudio para lupus eritematoso sistémico negativo; Ac anti $\mathrm{P}$ ribosomal $<1$ negativo. Una nueva resonancia de encéfalo seis días después del primer estudio mostró una lesión hiperintensa en la mayoría de las secuencias, excepto en T1, donde se vio hipointensa de bordes irregulares en cara interna del lóbulo occipital izquierdo, porción caudal del giro lingular y temporo-occipital ipsilateral; la angiorresonancia nuevamente con reporte de vasoespasmo bilateral de predominio izquierda de las cerebrales posteriores, la valoración por oftalmología se reporta normal sin datos de vasculitis retiniana. Egresa en buenas condiciones generales, asintomática. Gases arteriales normales, lactato $0.8 \mathrm{mmol} / \mathrm{L}$. Constantes vitales normales durante todo el internamiento.

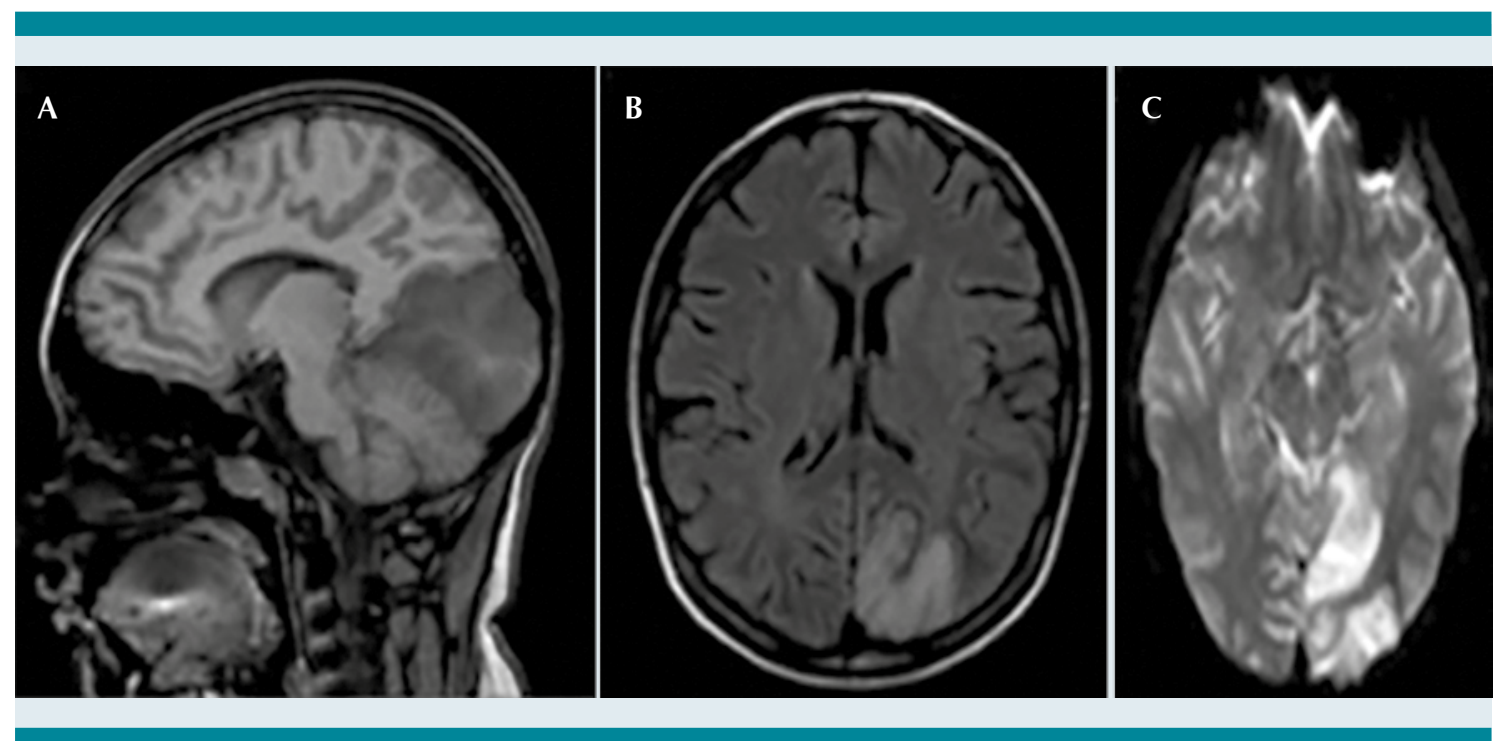

Figura 1. Primera resonancia magnética de encéfalo en secuencias A) T1 sagital, B) FLAIR axial, C) difusión axial donde se observa hipo-intensidad occipital izquierda así como hiperintensidad y restricción de señal de forma respectiva en la misma región. 
Reingresa a la unidad 72 horas después debido a cuadro de 12 horas de evolución con fosfenos, visión borrosa y cefalea frontal bilateral de intensidad 8/10 (escala visual análoga) y seguido de amaurosis bilateral. Una tomografía computarizada de cráneo a su llegada confirma la imagen previa de infarto izquierdo sin isquemia en región diferente a la descrita. Aparece taquicardia e hipotensión, así como fiebre de 39.3 grados centígrados. Gasometría arterial de ingreso con: pH 7.29, pO2 $64.4 \mathrm{mmHg}$, pCO2 $24.9 \mathrm{mmHg}$, HCO3 $14 \mathrm{mmol} / \mathrm{L}$, lactato $39.3 \mathrm{mmol} / \mathrm{L}$ con datos de choque sin foco infeccioso aparente. Se inicia terapia antibiótica. Persistencia con lactatos elevados en controles gasométricos promedio en $10.8 \mathrm{mmol} / \mathrm{L}$ con permanencia de fiebre, acidosis metabólica y datos de bajo gasto cardiaco que requirieron apoyo aminérgico en la unidad intensiva pediátrica.

Una nueva resonancia magnética en la unidad intensiva pediátrica mostró que persistía hiperintensidad mal definida en región occipital izquierda con extensión a región occipital centrolateral y contralateral con pérdida de relación de sustancias y restricción occipital bilateral en secuencia de difusión (Figura 2), los ácidos láctico y pirúvico se reportaron normales, así como un estudio inmunológico ac anti P-Ribosomal, ac antinucleosomas, ac antifosfolípidos, cardiolipina, B2 glicoproteína, fosfatidilserina, fosfatidilinositol, etc. Se inició terapia esteroidea con metilprednisolona a dosis de $1 \mathrm{~g} /$ día, hubo mejoría clínica posterior a 5 bolos.

Persistía la taquicardia supraventricular. Se trató con propanolol, sin cardiomiopatía ni otras alteraciones de conducción cardiaca. Se solicitó estudio molecular para MELAS, el cual se efectuó de forma privada (nanolab subrogado por ISSEMyM) a través de PCR y secuenciación directa de las regiones del gen MT-ND5 que incluye la mutación mas frecuente m.13513G>A y del gen MTL1 que incluye las mutaciones frecuentes $m .3271 t>C$ y $m .3252 A>G$. Las variantes normales (polimorfismo) descritas en la literatura o listadas en dbSNP (base de datos de polimorfismos y presentes en este estudio no son reportadas). Con lo cual se detectó la mutación $m .3271 T>C$ en heteroplasmia en el gen MT-TL1, sin detectar otras mutaciones en el estudio, lo cual es congruente con síndrome de MELAS causado por mutación en el gen MT-TL1. El tratamiento actual se basa en coenzima $\mathrm{Q}$, L-arginina y riboflavina.

\section{DISCUSIÓN}

La paciente tuvo datos clínicos iniciales de cefalea y visión borrosa, además de cambios en el comportamiento habitual y vómito, a esta sintomatología no se dio el valor clínico suficiente; sin embargo, en la descripción del síndrome según Lorenzoni P y sus colaboradores, la cefalea ocurre en la mayoría de los pacientes y se vuelve mucho más intensa durante la fase aguda del stroke-like, ${ }^{7}$ del mismo modo como se comenta en ese estudio son frecuentes las manifestaciones psiquiátricas así como alteraciones en los movimientos oculares. ${ }^{7}$

En nuestra paciente llaman la atención las manifestaciones clínicas visuales, debidas al territorio afectado en la circulación cerebral posterior. Los datos de imagen en nuestro caso son similares a lo descrito en la literatura de acuerdo con Xie y sus colaboradores, donde los hallazgos en la resonancia magnética en pacientes con MELAS han demostrado lesiones que afectan predominantemente la sustancia gris sin un territorio vascular específico las cuales son hiperintensas en T2 y FLAIR. ${ }^{8}$

Se ha especulado que en la fase aguda de episodios tipo stroke en MELAS, la acumulación local de lactato debido a la deficiencia o disfunción de enzimas oxidativas mitocondriales podría resultar en un cambio de las propiedades vascu- 


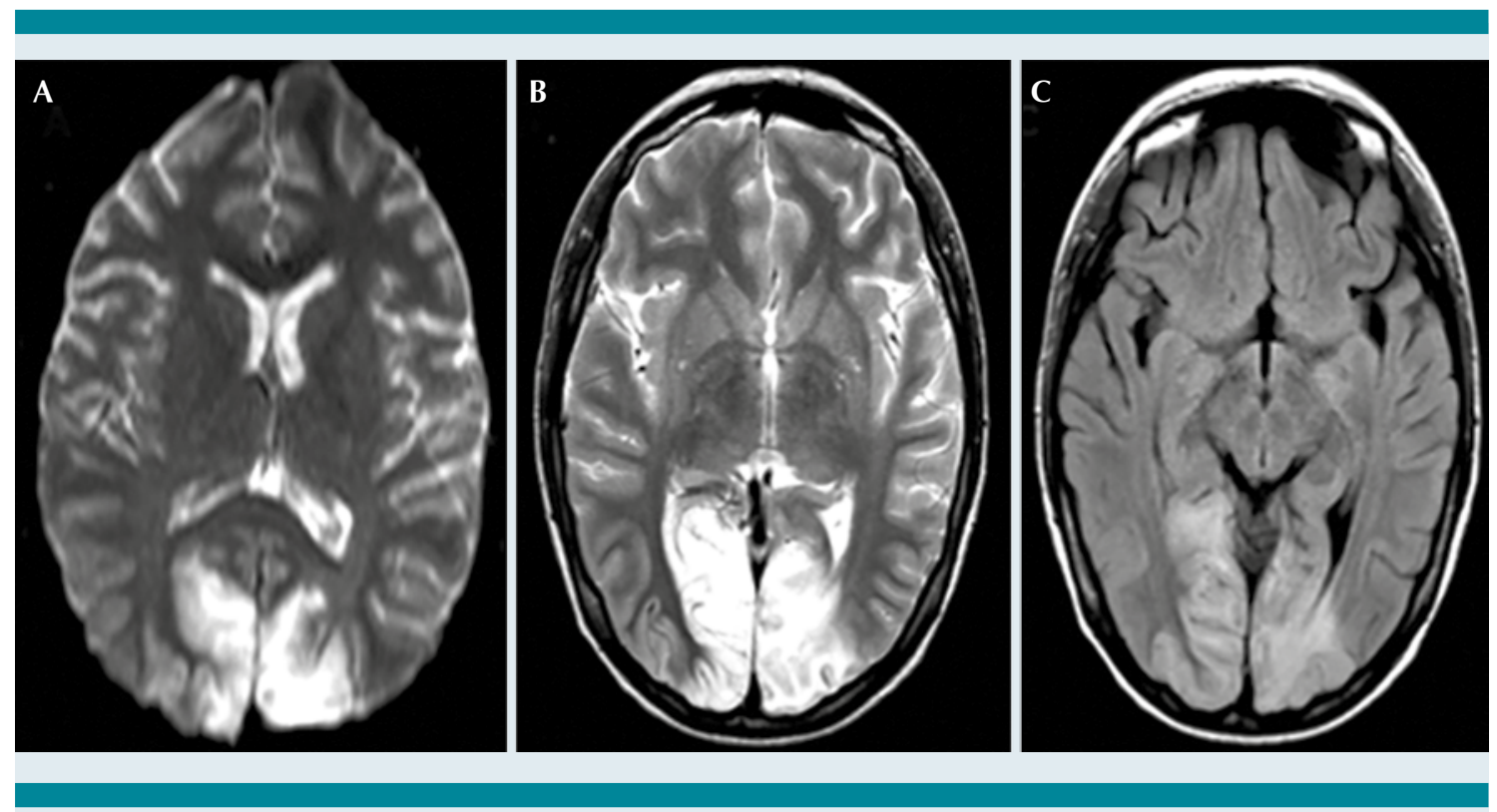

Figura 2. Resonancia magnética de encéfalo realizada en la unidad de cuidados intensivos en secuencias A) difusión axial, B) T2 axial, C) FLAIR axial donde se observa restricción en secuencia de difusión, hiper-intensidades en regiones occipitales bilaterales en secuencia T2 y FLAIR, respectivamente.

lares regionales, incluyendo datos de incremento en la permeabilidad capilar, inducir edema e hiperperfusión local. La hiperperfusión podría ser el resultado directo de la anormalidad en los mecanismos autorregulatorios vasculares en la angiopatía mitocondrial especialmente en las regiones corticales a causa de los abundantes vasos en estas zonas. ${ }^{8-11}$ Otras secuencias que se sugieren en el trabajo de Xie son la extracción de fracción de oxígeno, así como secuencia BOLD (blood-oxygen-level-dependent) como un complemento para el diagnóstico. ${ }^{8}$

Lo característico del caso es la presentación clínica tan severa en el segundo internamiento de nuestra paciente con manifestaciones sistémicas determinadas por acidemia e hiperlactatemia confirmada lo que a su vez comprometió seriamente el estado hemodinámico y ventilatorio de la paciente. Aparentemente sin factores que dispararan el desequilibrio ácido-base en ese momento y el estado de choque de probable ori- gen metabólico, estos datos se podrían equiparar con otros trabajos previamente descritos como el de Stenqvist y sus colaboradores donde reportan a una paciente joven con la misma mutación de nuestro caso con altos niveles de lactato (23.15 mmol/L) y un estado ácido severo con pH de 6.9 así como compromiso hemodinámico similar a lo que reportamos. ${ }^{2}$ Por otro lado, Ilama la atención la taquicardia supraventricular sin defecto estructural cardiaco que requirió el uso de beta bloqueadores, y que como se menciona en otros trabajos, no es común en pacientes con esta mutación.

Posterior al descubrimiento de la mutación A3243G, un segundo punto de mutación en la sustitución de T por C en el nucleótido en la posición 3271 se determino también en los pacientes con MELAS, convirtiéndose la mutación T3271C en la responsable de $7.5 \%$ de los casos aproximadamente, ${ }^{7}$ en nuestro medio la posibilidad de la determinación molecular resulta 
aún limitada sin embargo la realidad actual nos obliga a realizar la clasificación molecular de estas enfermedades con el fin de establecer un pronóstico más claro en cuanto a las comorbilidades asociadas a la enfermedad.

En cuanto al tratamiento, existen varias propuestas, se ha especulado que la L-arginina puede proteger las neuronas en la corteza de la muerte celular permanente ya sea mediante la mejoría de la microcirculación en el flujo sanguíneo regional del cerebro, la permeabilidad de la barrera hematoencefálica o bien interactuando en el acoplamiento entre el astorcito y la neurona, efecto similar causado por los esteroides los cuales se han utilizado junto con bolos de arginina en los episodios de stroke-like. ${ }^{9,10}$ Por otro lado, cabe señalar que algunos estudios han puesto en duda el uso de aspirina en estos pacientes ya que esta puede resultar tóxica para la mitocondria atenuando la movilización de protones, por lo que se ha sugerido que la aspirina no debe utilizarse en el tratamiento de pacientes con mitocondropatías. ${ }^{10}$

A diferencia de lo expresado en trabajos previos actualmente se sabe que la realización de ejercicio físico regular puede mejorar la resistencia al ejercicio en estos pacientes. El entrenamiento de fondo puede inducir biogénesis mitcondrial, mientras que el entrenamiento físico de resistencia puede producir transferencia de estructuras mitocondriales normales a células satélite del músculo maduro con lo cual se podría disminuir la heteroplasmia en la mutaciónes. ${ }^{12}$

Desde las primeras descripciones formales se ha utilizado coenzima Q-10 como uno de los manejos base en la enfermedad, la coenzima Q-10 facilita la transferencia de electrones de los complejos I y II al complejo III de la cadena respiratoria, estabilizando los complejos y brindando un efecto antioxidante protector. ${ }^{13}$ Con lo cual se han mostrado beneficios sobre todo en cuanto a debilidad muscular, actualmente las presentaciones son variadas y es de alto costo sin embargo sigue constituyendo uno de los manejos soportes en los pacientes con mitocondropatías.

A pesar de los datos publicados sobre esta entidad el conocimiento entre los pediatras aún es limitado, y es importante determinar algunos medicamentos que podrían alterar la evolución de los pacientes como es el valproato que podría incrementar la acidosis mitocondrial e incluso provocar crisis en estos pacientes, otros fármacos antiepilépticos que podrían afectar el metabolismo mitocondrial incluyen: fenobarbital, carbamazepina, fenitoína, oxcarbazepina, etosuximida, zonisamida, topiramato, gabapentina y vigabatrina. ${ }^{14,15}$ Con el fin de permitirle a los pacientes un mejor control que pueda prevenir recaídas en la evolución de su enfermedad.

Por otro lado, vale la pena considerar la asociación de otras entidades que pueden presentar manifestaciones tipo stroke-like las cuales se pensaban con anterioridad eran exclusivas de MELAS, estas posibilidades incluyen: epilepsia mioclónica con fibras rojas rasgadas, síndrome de Kearns-sayre, síndrome de Leigh, neuropatía óptica hereditaria de Leber's, oftalmoplejía externa progresiva, deficiencia de propionil-CoA carboxilasa, en pacientes con mutaciones del gen POLG1, en el síndrome triple $\mathrm{H}$ y en la ataxia espinocerebelosa mitocondrial. ${ }^{16-17}$ Por lo que el abordaje del paciente con episodios considerados como stroke-like nos obliga a realizar una búsqueda imperativa de manifestaciones que pudieran compaginarse con algunas de las patologías comentadas.

La biopsia muscular constituye un método importante en el escrutinio de la enfermedad, habitualmente en el tejido se puede observar la presencia de fibras rojas rasgadas las cuales resultan positivas a la tinción de Gomori o bien la presencia de fibras azules rasgadas resultantes de 
la tinción de succinato deshidrogenasa, siendo una característica que en MELAS estas fibras se tiñen de forma positiva ante el citocromo $C$ oxidasa a diferencia de otros trastornos como en el síndrome de Kearns-Sayre y epilepsia mioclónica con fibras rojas rasgadas en la que las fibras no reaccionan al citrocromo $\mathrm{C}$ oxidasa. Otro de los datos característicos (no patognomónicos) en MELAS es la abundancia de mitocondrias en el músculo liso y células endoteliales. ${ }^{18,19}$ Por lo anterior, la realización de una biopsia muscular en estos pacientes deberá ser considerada ante la sospecha diagnóstica la facilidad del procedimiento considerando la necesidad de que el tejido sea valorado por expertos patólogos en estas entidades.

\section{CONCLUSIONES}

Los episodios de stroke-like en pacientes pediátricos se han presentado con mayor frecuencia en los últimos años, los abordajes incluyen la neuroimagen, perfiles de coagulación e inmunológicos que tengan a bien lograr determinar la causa del accidente vascular, sin embargo es factible que el advenimiento de nuevas técnicas moleculares cada día disponibles con mayor facilidad para su realización se conviertan en estudios iniciales y no únicamente como pruebas confirmatorias, lo cual favorece establecer de manera prematura el problema y establecer los factores pronósticos.

Resulta importante del mismo modo establecer la implicación multiorgánica en estos pacientes, lo cual depende del efecto provocado por el tipo de mutación que presentan, obligándonos a un manejo multidisciplinario con el fin de un mejor seguimiento individualizando cada caso.

El manejo suplementario a base de Co-enzima Q10, L-arginina y riboflavina entre otros bioelementos; acompañados de actividad física y un seguimiento cercano son algunas de las estrategias que se pueden realizar para evitar factores precipitantes de estos episodios; las cuales pueden llevarse a cabo en hospitales de segundo nivel de atención; de ahí la importancia de difundir este tipo de entidades clínicas.

La secuenciación de genes debe convertirse ya en una herramienta que nos permita determinar el comportamiento de diversas entidades neurológicas en nuestra población con el fin de poder caracterizar las presentaciones clínicas en el caso de las enfermedades con alta sospecha genética o en este caso mitocondrial.

\section{REFERENCIAS}

1. Goodfellow J, Dani K, Stewart W, Santosh C, McLean J, Mulhern S et al. Mitochondrial myopathy, encephalopathy, lactic acidosis and stroke-like episodes: an important cause of stroke in young people. Postgraduate Medical Journal. 2012;88(1040):326-334.

2. Stenqvist L, Paetau A, Valanne L, Suomalainen A, Pihko H. A Juvenile Case of MELAS with T3271C Mitochondrial DNA Mutation. Pediatric Research. 2005;58(2):258-262.

3. Yatsuga S, Povalko N, Nishioka J, Katayama K, Kakimoto N, Matsuishi T et al. MELAS: A nationwide prospective cohort study of 96 patients in Japan. Biochimica et Biophysica Acta (BBA) - General Subjects. 2012;1820(5):619-624.

4. Mitani T, Aida N, Tomiyasu M, Wada T, Osaka H. Transient ischemic attack-like episodes without stroke-like lesions in MELAS. Pediatric Radiology. 2013;43(10):1400-1403.

5. Brisca G, Fiorillo C, Nesti C, Trucco F, Derchi M, Andaloro A et al. Early onset cardiomyopathy associated with the mitochondrial tRNALeu(UUR) 3271T>C MELAS mutation. Biochemical and Biophysical Research. Communications. 2015;458(3):601-604.

6. Yu N, Zhang $Y$, Zhang $K$, Xie $Y$, Lin $X$, Di Q. MELAS and Kearns-Sayre overlap syndrome due to the mtDNA $m$. A3243G mutation and large-scale mtDNA deletions. e Neurological Sci. 2016;4:15-18.

7. Lorenzoni P, Werneck L, Kay C, Silvado C, Scola R. When should MELAS (Mitochondrial myopathy, Encephalopathy, Lactic Acidosis, and Stroke-like episodes) be the diagnosis? Arq Neuro-Psiquiatr. 2015;73(11):959-967.

8. Xie S. MR OEF Imaging in MELAS. Methods in Enzymology. 2014;:433-44.

9. Kitamura M, Yatsuga S, Abe T, Povalko N, Saiki R, Ushijima $\mathrm{K}$ et al. I- Arginine intervention at hyper-acute phase protects the prolonged MRI abnormality in MELAS. Journal of Neurology. 2016;263(8):1666-1668. 
10. Fryer R, Bain J, De Vivo D. Mitochondrial Encephalomyopathy Lactic Acidosis and Stroke-Like Episodes (MELAS): A Case Report and Critical Reappraisal of Treatment Options. Pediatric Neurology. 2016;56:59-61.

11. Li R, Xiao H, Lyu J, J.J. Wang D, Ma L, Lou X. Differential diagnosis of mitochondrial encephalopathy with lactic acidosis and stroke-like episodes (MELAS) and ischemic stroke using 3D pseudocontinuous arterial spin labeling. J Magn Reson Imaging. 2016. doi: 10.1002/jmri.25354

12. El-Hattab A, Adesina A, Jones J, Scaglia F. MELAS syndrome: Clinical manifestations, pathogenesis, and treatment options. Molecular Genetics and Metabolism. 2015;116(1-2):4-12.

13. Glover E, Martin J, Maher A, Thornhill R, Moran G, Tarnopolsky M. A. randomized trial of coenzyme Q10in mitochondrial disorders. Muscle \& Nerve. 2010;42(5):739-748.

14. Lam C, Lau C, Williams J, Chan Y, Wong L. Mitochondrial myopathy, encephalopathy, lactic acidosis and stroke-like episodes (MELAS) triggered by valproate therapy. European Journal of Pediatrics. 1997;156(7):562-564.
15. Finsterer JZarrouk Mahjoub S. Mitochondrial toxicity of antiepileptic drugs and their tolerability in mitochondrial disorders. Expert Opinion on Drug Metabolism \& Toxicology. 2011;8(1):71-79.

16. Morin C, Dubé J, Robinson B, Lacroix J, Michaud J, De Braekeleer $\mathrm{M}$ et al. Stroke-like episodes in autosomal recessive cytochrome oxidase deficiency. Annals of Neurology. 1999;45(3):389-392.

17. Vallée L, Fontaine M, Nuyts J, Ricart G, Krivosic I, Divry $P$ et al. Stroke, hemiparesis and deficient mitochondrial $\beta$-oxidation. European Journal of Pediatrics. 1994;153(8):598-603.

18. DiMauro S. Mitochondrial encephalomyopathies: problems in pathogenesis. Electroencephalography and Clinical Neurophysiology. 1997;103(1):24-25.

19. Filosto $M$, Tomelleri G, Tonin P, Scarpelli M, Vattemi G, Rizzuto N, Padovani A, Simonati A. Neuropathology of mitochondrial diseases. Biosci Rep. 2007;27:23-30 